In the Netherlands the GP is the main care provider, also for sexual health. We investigated the trend in HIV-related consultations and testing practises in general practise in the last 2 decades.

Methods Within a nationally representative Dutch Sentinel General Practice Network we analysed HIV-related consultations from 1988-2009 using a questionnaire, in which patient's characteristics, the reason for consulting the GP, interventions and test results were recorded. Trends over time were calculated by multilevel analysis.

Results Time trend analyses show an increasing trend in HIVrelated consultations and in the total number of HIV tests per 10,000 registered patients. Mean number of consultations doubled from 7 in 1988 to 14 consultations/10.000 population in 2009. Over the whole period, the number of HIV-related consultations was significantly higher in urban areas. The proportion of people high at risk, men who have sex with men (MSM) decreased. The proportion of HIV related consultations initiated by the GP increased from $11 \%$ in 1988 to $23 \%$ in 2009.

Conclusion In this 20-year period the policies around HIV testing changed drastically. HIV-related consultations, as well as provider initiated testing in general practise in the Netherlands increased, however slowly and mainly in low risk groups. Testing rates remain low. To prevent undiagnosed and late presentation of HIV infection, GP need to be more pro-active. New and innovative avenues, like opt-out and indicator-based testing, are needed in primary care as many test-opportunities among high risk groups and in high risk areas are missed.

\section{P5.031 CERVICAL CANCER AND STI SCREENING: A MUST IN THE ERA OF HIV/AIDS; INCREASING ACCESS FOR HIV INFECTED WOMEN THROUGH COMMUNITY OUTREACH SCREENING PROGRAMMES}

doi:10.1136/sextrans-2013-051184.1076

J F Acio, E Schouten, M Arinaitwe, N Lukoda, D Akurut, S Kellerman, E Ssemafumu. Management Sciences for Health, Inc. Joint Clinical Research Centre, Kampala, Uganda

Background Cervical cancer $(\mathrm{CaCx})$ is the most frequently diagnosed cancer in Ugandan women. Forty five out of 100,000 women die annually due to limited availability of information, screening and treatment facilities, and poor health seeking habits. Over $80 \%$ of women present when in advanced stages. The risk of $\mathrm{CaCx}$ and STIs is doubled among HIV-infected women. STAR-E project instituted an integrated community outreach screening programme targeting WLHIV to accelerate early diagnosis, enhance timely referral for treatment and provide prevention education.

Methods Between March 2011 to April 2012, we reviewed records in 12 HIV care clinics to identify WLHIV at risk of STIs and CaCx. Key health workers were trained in STI diagnosis, and VIA/VILI screening methods. 50 PLHIV mobilisers were identified and oriented on risk factors, prevention, and early warning signs of $\mathrm{CaCx}$ and common STIs. During home visits, treatment support meetings and clinic days, and using pre-designed referral forms containing individual questionnaire they sensitised and mobilised community, and made referrals to lower level health centres where screening camps were set.

Results A total of 3500 WLHIV were sensitised and referred for STI and $\mathrm{CaCx}$ screening. 3450 (98.5\%) were screened for the first time; $3405(98.7 \%)$ tested VIA negative; $45(1.3 \%)$ tested VIA positive and were referred for cryotherapy. of all women screened, 342 (9.9\%) presented with different STIs, and were treated. 238 (69.6\%) notified their partners who also accessed treatment. Only $20 \%$ of the women had heard about $\mathrm{CaCx}$ or sought help for any genital infections.

Conclusion Integrated outreach screening programmes are an important access point for people at high risk for both STIs and $\mathrm{CaCx}$. It allows for not only the benefit of treating the STI, or identifying precancerous lesions, but for prevention education, identifying HIVinfected persons in need of care, and partner notification for STIs.

\section{P5.032* DO FAST-TRACK ASYMPTOMATIC SCREENING PATHWAYS FOR MEN WHO HAVE SEX WITH MEN (MSM) LEAD TO MISSED OR DELAYED DIAGNOSES?}

\author{
doi:10.1136/sextrans-2013-051184.1077
}

${ }^{1} \mathbf{M}$ Bains, ${ }^{1} \mathrm{~A}$ Collister, ${ }^{2} \mathrm{R}$ Jackson, ${ }^{2} \mathrm{E}$ Clarke, ${ }^{2} \mathrm{R}$ Patel. ${ }^{1}$ University of Southampton, Southampton, UK, ${ }^{2}$ Royal South Hants Hospital, Southampton, UK

Background Within sexual health clinics in the UK MSM are usually examined with clinician-taken swabs. In an effort to increase access and encourage regular screening, a minority of clinics across the UK are now using a fast-track asymptomatic pathway for MSM with patient-taken swabs. However the consequences of not examining asymptomatic MSM remain unclear.

Aim Would the introduction of a fast-track asymptomatic screening pathway for MSM lead to missed diagnoses or delayed or withheld treatment?

Method This was a retrospective case note review of all MSM attending our UK level 3 sexual health clinic between July 2011 and July 2012. Clinical findings, diagnoses and treatments given to asymptomatic MSM were analysed further.

Results 476 of 920 MSM attendances were asymptomatic presentations, of which 21 (4.4\%) had positive findings on examination. Perianal abnormalities were detected in 1\% (peri-anal warts $n=4$, skin tags $n=1)$. Genital abnoramlities were penile warts $(n=2)$, epididymal cyst $(n=1)$, variocele $(n=1)$, unretractable foreskin $(\mathrm{n}=1)$, and 3 dermatological finding (dry meatus, abrasion on penis and penile erythema). Contact bleeding on proctoscopy was found in $2(0.4 \%)$ patients, and $7(1.5 \%)$ cases of non-specific urethritis (NSU) were identified. All 9 of these cases were negative for Chlamydia trachomatis and Neisseria gonorrhoeae.

Conclusion Had the asymptomatic MSM attendances not involved examination, no significant clinical diagnoses would have been missed, or any treatment delayed or withheld. These findings suggest a fast-track asymptomatic screening pathway for MSM with patient-taken swabs could be introduced into UK sexual health clinics without concern. This in turn would increase sexual health screening for this high risk group. However consideration of missing perianal abnormalities in this group is needed as it may be of greater importance when compared to heterosexual males.

\section{P5.033 BRINGING HIV TESTING TO THE PEOPLE- BENEFITS OF MOBILE UNIT TESTING IN LIMA, PERU, 2007-2009}

doi:10.1136/sextrans-2013-051184.1078

1,2M C Lipsitz, 'E R Segura, ${ }^{2} \mathrm{C}$ Anton, ${ }^{2} \mathrm{~J}$ Castro, ${ }^{1} \mathrm{~J}$ L Clark, ${ }^{1} \mathrm{~J}$ E Lake, ${ }^{2} \mathrm{R}$ Cabello. ${ }^{1}$ University of California, Los Angeles, Los Angeles, CA, United States; ${ }^{2}$ Vía Libre, Lima, Peru

Background Mobile unit (MU) HIV testing is an alternative method of providing healthcare access. We compared demographic and behavioural characteristics, HIV testing history, and HIV prevalence between people seeking testing at a MU vs. fixed community clinic (FC) in Lima, Peru.

Methods Our analysis included men and transwomen (TW) in Lima $\geq 18$ years old seeking HIV testing at their first visit to a community-based clinic's MU or FC from Oct. 2007-Nov. 2009. Behavioral characteristics, including HIV testing history, and HIV serostatus of the following populations were compared in MU vs. FC attendees: TW, men who have sex with men (MSM), and heterosexually self-identified men who have sex with men and women (MSMW).

Results A greater percentage of $\mathrm{MU}$ attendees self-identified as transgender ( $8 \%$ vs. $3 \%$ FC, $p<0.05$ ) or heterosexual $(52 \%$ vs. $15 \%$ $\mathrm{FC}, \mathrm{p}<0.05)$. MU and FC sites attracted similar proportions of MSMW (52\% MU vs. 57\% FC). MU attendees were more likely to 